\title{
Vocational Rehabilitation with or without Work Module for Patients with Chronic Musculoskeletal Pain and Sick Leave from Work: Longitudinal Impact on Work Participation
}

\author{
Timo T. Beemster ${ }^{1,2,3}$. Coen A. M. van Bennekom ${ }^{2,3}$. Judith M. van Velzen ${ }^{2,3} \cdot$ Monique H. W. Frings-Dresen $^{3}$. \\ Michiel F. Reneman ${ }^{1}$
}

Published online: 6 May 2020

(c) The Author(s) 2020

\begin{abstract}
Purpose To study the longitudinal relationship between interdisciplinary vocational rehabilitation (VR) with and without additional work module on work participation of patients with chronic musculoskeletal pain and sick leave from work. Methods Retrospective longitudinal data retrieved from care as usual in seven VR centers in the Netherlands was used. The VR program without work module consisted of multi-component healthcare (physical exercise, cognitive behavioral therapy, education, relaxation). The other program with additional work module (VR+) included case management and a workplace visit. Generalized estimating equations using binary logistic was applied. The dependent variable was work participation (achieved/not achieved) on discharge and 6-months follow-up. Independent variables were type of intervention, return to work expectation, sick leave duration, working status, job strain, and job dissatisfaction. Results Data from $\mathrm{N}=470$ patients were analyzed, of which $26 \%$ received VR and $74 \% \mathrm{VR}+$. Both programs increased work participation at 6-months followup (VR 86\%, VR+87\%). The crude model showed a significant longitudinal relationship between type of intervention and work participation in favor of $\mathrm{VR}+(\mathrm{OR} 1.8, \mathrm{p}=0.01)$. The final model showed a non-significant relationship on discharge $(\mathrm{OR} 1.3, \mathrm{p}=0.51)$ and a significant relationship on 6-months follow-up in favor of VR+(OR 1.7, p=0.04). RTW expectation was a significant confounder in the final model on discharge and 6-months follow up (OR 3.1, $\mathrm{p}=0.00)$. Conclusions Both programs led to increased work participation. The addition of a work module to the VR program lead to a significant increase in odds of work participation at 6-months follow-up.
\end{abstract}

Keywords Chronic pain · Observational study $\cdot$ Occupational therapy $\cdot$ Biopsychosocial $\cdot$ Multidisciplinary

\section{Introduction}

Chronic musculoskeletal pain (CMP) affects quality of life, disability, and work $[1,2]$. Workers with CMP have high rates of absenteeism and presenteeism (presenteeism: the phenomenon of people still turning up at their jobs, despite

Michiel F. Reneman

m.f.reneman@umcg.nl

1 University Medical Center Groningen, Department of Rehabilitation Medicine, University of Groningen, Haren, P.O. Box 30.002, 9750 RA Groningen, The Netherlands

2 Department of Research and Development, Heliomare Rehabilitation Center, Wijk aan Zee, The Netherlands

3 Amsterdam UMC, Coronel Institute of Occupational Health, Amsterdam Public Health Research Institute, University of Amsterdam, Amsterdam, The Netherlands complaints and ill health that should prompt rest and absence from work [3]), with productivity losses equivalent to $1.6 \%$ of Gross Domestic Product for the Netherlands [4]. Thus, the main goal of interventions for patients with CMP and productivity loss from work is to increase work participation. Several reviews have shown that interdisciplinary vocational rehabilitation (VR) programs are effective in realizing this goal [5-7].

There is large variation in the content of VR programs [5-8]. A recent review recommended that effective VR programs should encompass the following three domains: 1 . health-focused (i.e., health services intervention subcategories such as graded activity/exercise, cognitive behavioral therapy [CBT], work-hardening), 2. service coordination (i.e., improving communication within the workplace or between the workplace and the healthcare providers), and 3 . work modification (i.e., modified duties, modified working 
hours, supernumerary replacements, ergonomic adjustments, or other worksite adjustments) (Box 1) [5]. The same review also mentioned that a multi-domain intervention including components in at least two of the three domains mentioned, can help reduce lost time from work for CMP-related conditions [5].

The review mentioned above and other studies on this topic mainly consist of RCT studies in which multi-domain programs were compared with usual care $[5,6,9]$ or with single component programs from the health-focused domain, such as graded activity/physical exercise $[6,8,10]$, or education [6]. Other RCTs showed positive results of the additional effect on work participation of patients with acute and subacute low back pain when the work-related component was added to a multidisciplinary, health-focused program [11-14]. However, little evidence is available about the additional increase in effect on work participation when components from the work-related domains (i.e., service coordination and work modifications; Box 1) are added to a multi-component health-focused program conducted for patients with CMP. The addition of a focused work module appears not part of standard care for patients with CMP in most industrialized countries. However, the evidence concerning this niche is contradictory.

On the one hand, an RCT study conducted in Norway in patients with neck and back pain found no significant differences in work participation between the group who took part in a multidisciplinary program (i.e., multi-components from the health-focused domain) that included work-focused components and a group who only took part in a multidisciplinary program [15]. On the other hand, a retrospective cohort study conducted in Canada showed that a multidisciplinary (i.e., multi-components from the health-focused domain) pain program that included return to work coordination had 3.4 higher odds of a return to work compared with a multidisciplinary program without coordination [16].

In summary, while the evidence on the overall effectiveness of VR is robustly positive, the evidence concerning the content of VR is contradictory. In the present study, we analyzed the difference in work participation of patients who were referred to multi-component health-focused VR program with or without an additional work module in clinical practices in the Netherlands (VR+and VR respectively).

The research question of this study was: Are patients with CMP who are on sick leave from work more likely to participate in work if they take part in a $\mathrm{VR}+$ program compared with patients who only take part in a VR program? Based on recommendations from various systematic reviews to include work domains in VR to achieve successful work participation [5, 6, 9, 17], we hypothesized that patients who took part in the VR+ program would have higher odds of participating in work compared to patients who only took part in the VR program.

\section{Methods}

The Strengthening the Reporting of Observational Studies in Epidemiology (STROBE) checklist was used in the design and reporting of this study [18].

\section{Design, Setting, and Procedure}

A retrospective cohort study was conducted, with data collected from November 2014 to July 2018 by seven rehabilitation centers located throughout the Netherlands. These seven centers all offered interdisciplinary VR for workers with CMP who were hampered in their work participation. Patients were referred to the VR program by their occupational physician, general physician, rehabilitation physician, medical specialist, or others. Before entering the VR program, patients completed web-based questionnaires (T0) and underwent a multidisciplinary (MD) screening performed by an MD team consisting of a rehabilitation physician, psychologist, physical therapist, and vocational specialist. After the MD screening, the team and patient decided whether a VR+ program was appropriate or not (criteria, see [19]). Before VR+started, the employer of every patient was asked to reimburse the additional work module (€1200), which was a condition of the patient participating in the $\mathrm{VR}+$ program. VR was reimbursed by the healthcare insurer. Apart from the additional work module, patients of both programs participated as one group. Patients received web-based questionnaires at discharge (T1) and at 6-months follow-up (T2). If patients did not complete the T0-2 questionnaires within a week, they received a reminder by email.

\section{Participants}

Working age individuals (18-65 years) with subacute or chronic musculoskeletal pain and reduced work participation (full or part-time sick leave) who were referred to vocational rehabilitation and who underwent a vocational rehabilitation program (VR+or VR) between September 2014 and October 2017 participated in this study. Patients were excluded if they had no paid work, if they were not able to complete questionnaires in Dutch, or if they did not grant informed consent. The Medical Ethical Committee of the Academic Medical Center, Amsterdam, the Netherlands, authorized this study and decided that a full application was not required (Number W18_194). Participation in the study was voluntary, all participants provided informed consent, and answers were processed anonymously. 
Box 1 Intervention components in rehabilitation treatments

Health-focused interventions These interventions facilitate the delivery of health services to the injured worker either in the workplace or in settings linked to the workplace (e.g., visits to healthcare providers initiated by the employer/workplace). Specific health services intervention subcategories for which evidence synthesis was conducted include; graded activity/ exercise, cognitive behavioural therapy, work hardening and multi-component health-focused interventions (which often included the above elements as well as: medical assessment, physical therapy, psychological therapy, occupational therapy)

Service coordination interventions These interventions were designed to better coordinate the delivery of, and access to, services to assist RTW within and involving the workplace. Coordination involves attempts to improve communication within the workplace or between the workplace and the healthcare providers. Examples are development of RTW plans, case management and education and training

Work modification interventions These interventions alter the organization of work or introduce modified working conditions. Examples are: workplace accommodations such as provision of modified duties, modified working hours, supernumerary replacements, ergonomic adjustments or other worksite adjustments

Multi-domain interventions These interventions had multiple intervention components and included at least two of the three above intervention domains [e.g., interventions that involved graded activity in the workplace (health-focused domain) in addition to modified working conditions (work modification domain)]

Text obtained from Cullen et al. [4]

\section{Context}

When an employee is sick-listed in the Netherlands, both the employee and employer are responsible for the work participation process during the first 2 years of sick leave. According to the Dutch Gatekeeper Improvement Act, the employer must provide wage replacement and modified work during this 2-year period [20].

\section{Interventions}

\section{Vocational Rehabilitation (VR)}

The vocational rehabilitation (VR) program was an interdisciplinary group-based program that consisted of multi-components from the health-focused domain. They included general exercise therapy based on principles of graded activity (total $\sim 60 \mathrm{~h} ; 30 \times 2 \mathrm{~h}$ ), CBT (total $7.5 \mathrm{~h} ; 15 \times 0.5 \mathrm{~h}$ ), group education (total $\sim 15 \mathrm{~h} ; 15 \times 1 \mathrm{~h}$ ), and relaxation (total $\sim 7.5 \mathrm{~h}$; $15 \times 0.5 \mathrm{~h}$ ). There were two evaluation moments with the patient: one mid-evaluation after seven weeks and one end evaluation at discharge. A report from these two evaluation moments was sent to the patient. The MD team consisted of a physician, physiotherapist, and a psychologist. The program lasted fifteen weeks (total $\sim 90 \mathrm{~h}$ ) with two 3.5 to $4 \mathrm{~h}$ sessions per week. More information about the content of the VR program can be found in the study protocol paper [21].

\section{Vocational Rehabilitation + Work Module (VR+)}

The vocational rehabilitation + work module $(\mathrm{VR}+)$ program was an interdisciplinary group-based program that consisted of the same health-focused components as the VR program, but was extended with a work module. The work module consisted of case management and a workplace visit (total of $\sim 10 \mathrm{~h}$ ), and was executed by an RTW coordinator. The case management involved discussion of work-related problems, the design and discussion of the progress of a work participation plan, and the provision of information about work-related legislation. The company visit included communication between the patient, the RTW coordinator, and the employer with the goal of discussing and resolving barriers to and facilitators of work participation, as well as discussing a work participation plan. A workplace inspection with possible advice for ergonomic adjustment was also part of the workplace visit. There were two evaluation moments with the patient: one mid-evaluation after seven weeks and one end evaluation at discharge. A report of these two evaluation moments was sent to the patient and his/her employer and occupational physician. If necessary, the evaluation reports were discussed with the employer and/or occupational physician. The MD team consisted of a physician, physiotherapist, psychologist, and an RTW coordinator. The program lasted fifteen weeks (total $\sim 100 \mathrm{~h}$ ) with two 3.5 to $4 \mathrm{~h}$ sessions per week. An outline of the content and dosage of the modules of the VR+ program are described in the study protocol paper [21].

\section{Measures}

\section{Dependent Variable: Work Participation}

Work participation was assessed using the working status item of the imta Productivity Cost Questionnaire-Vocational Rehabilitation version (iPCQ-VR) [22]. Working status was assessed with the question: "Are you working full-time at this moment?" with the answer categories: "Yes," "No, I am partly at work," and "No, I am on 100\% sick leave." In the case of patients being partly at work, there was an 
additional question: "How many hours are you working per week at the moment?" For the aim of this study, the working status and hours working per week items were first converted into a continuous variable of "hours working per week." In a second step, the change in working hours per week was calculated by subtracting working hours per week at T1/T2 from the working hours per week at T0. In a final step, the working hours per week difference was dichotomized into "Achieved work participation" for those who worked at least one hour or more per week at T1/T2 compared to T0, and "Not achieved work participation" for those who worked the same working hours per week or less at T1/T2.

\section{Independent Variables}

The fixed independent variable in this study was type of intervention $(\mathrm{VR}+/ \mathrm{VR})$. The other independent variables selected were potentially confounders of the outcome of "work participation." The independent variables of this study were clustered into biopsychosocial characteristics [23]: demographic, psychological, disorder-related, and work-related. Hereafter, we briefly describe the content and score ranges of the independent variables selected and used in this study. A detailed description and clinometric properties of the questionnaires included can be found elsewhere $[21,22,24]$.

\section{Demographic Characteristics}

The following demographic characteristics were included: age [25-28], gender [16, 26-29], and level of education [27, 30-33]. Age was dichotomized based on the median. Level of education was divided into three categories: "low" (including primary school, lower vocational education, and lower secondary school), "medium" (including intermediate vocational education and upper secondary school), and "high" (including upper vocational education or university) [30].

\section{Psychological Variables}

The following psychological characteristic was used: job-related illness behavior [30, 34, 35], was measured with a subscale from the Work Reintegration Questionnaire (WRQ) [34, 35]. The subscale consist of multiple statements which are answered on a 4-point Likert scale $(1=$ disagree, $2=$ somewhat agree, $3=$ quite agree, $4=$ completely agree). The illness behavior scale ranges from 10 to 40 and was dichotomized, with scores above 34 referring to high illness behavior [34].

\section{Disorder-Related Characteristics}

The following disorder-related characteristics were used: duration of complaints $[16,36]$, pain intensity $[25,27$, $28,37]$, widespread pain [26, 27, 38], level of disability $[25,27,39,40]$, and perceived health [27, 28]. Duration of complaints was dichotomized into "subacute" (duration of complaints 3 to 6 months) and "chronic" (more than six months) complaints [36]. Pain intensity was assessed on a 11-point Likert scale, as the mean pain score in the preceding week, where 0 denoted no pain and 10 denoted worst possible pain. Pain intensity was dichotomized into "high pain score" (score of $\geq 7$ ) versus "medium/low pain score" (score of $\leq 6$ ) [2]. Widespread pain was dichotomized into "yes" or "no." Widespread pain was defined as "yes," if pain in the upper extremities (arm, hand, or wrist), lower extremities (hip, knee, ankle, or foot) and axial skeletal pain (back) was present [41].

Level of disability was measured with the Pain Disability Index (PDI) [42], which is a 7-item questionnaire that measures self-reported pain-related disability. The PDI measures seven dimensions: family/home responsibilities, recreation, social activity, occupation, sexual behavior, self-care, and life support activity on a 0-10 scale ( 0 denotes "no disability" and 10 denotes "maximum disability"). Total scores range from 0 to 70 , with higher scores reflecting higher level of disability. The level of disability score was dichotomized based on the median. Perceived health was assessed with a single health status item obtained from the RAND-36 [43, 44]: "What do you think about your health in general?," with five answer categories, ranging from "excellent" to "bad." Perceived health was dichotomized into good health ("excellent," "very good," and "good") and moderate health ("moderate," "bad").

\section{Work-Related Characteristics}

The following work-related characteristics were used: $R T W$ expectation [27-30, 37, 45-47], sick leave duration [26, 27, 48, 49], working status [25, 27, 40, 50], job strain [32], and job dissatisfaction $[29,51]$. RTW expectation was assessed on a $0-10$ scale, with patients rating the certainty that they will be working in six months, where 0 represents "Not at all certain" to 10 "Extremely certain." We dichotomized this item into negative RTW expectancy (score 0-5) and positive RTW expectancy (score 6-10). Sick leave duration was assessed with the sick leave long item of the iPCQ-VR questionnaire [22]. We dichotomized this item into longterm sick leave or not ("yes" = absenteeism for six weeks or more; "no"= absenteeism for less than six weeks). The decision to consider a period of 6 weeks' sick leave in this study was based on Dutch social security legislation [52]. 
Working status was assessed with the working status item of the iPCQ-VR [22]. We dichotomized this item into "full sick leave" and "part-time sick leave." Job strain and job dissatisfaction were measured with two subscales of the WRQ, which were dichotomized based on norm scores [31]. The job strain scale ranges from 7 to 28 and was dichotomized, with scores above 17 referring to high job strain. The job dissatisfaction scale ranges from 12 to 48 and was dichotomized, with scores above 30 referring to high job dissatisfaction.

\section{Statistical Analyses}

Generalized estimating equations (GEE) analyses were applied. The analyses were performed in four steps. In the first step, a crude model was run with work participation (achieved/not achieved) as the dependent variable and type of intervention (VR/VR+) as the independent variable. In the second step, time and interaction of time and type of intervention was added to the crude model. In step three, we examined whether confounding variables were present. The model from step 2 was used, with as the reference for time: 6-months follow-up. If the regression coefficient of type of intervention increased or decreased $\geq 10 \%$, we considered the independent variable as a confounder. Based on the available evidence, we assumed a priori that RTW expectation [27-30, 37, 45-47], work status [25, 27, 40, $50]$, and sick leave duration [26, 27, 48, 49] were potential confounders. In the fourth and final step, the final model was run, consisting of type of intervention, time, interaction time*intervention type and confounding variables. We report odds ratios, 95\% confidence intervals of odds ratios, and p-values. All analyses were performed using SPSS Statistics for Windows, version 25.0 (2015), IBM Corp., Armonk, NY.

To visualize the relationship between type of intervention and the dependent variable work participation, working status proportions were provided for each time point (baseline, discharge, 6-months follow-up) separated for VR and VR+.

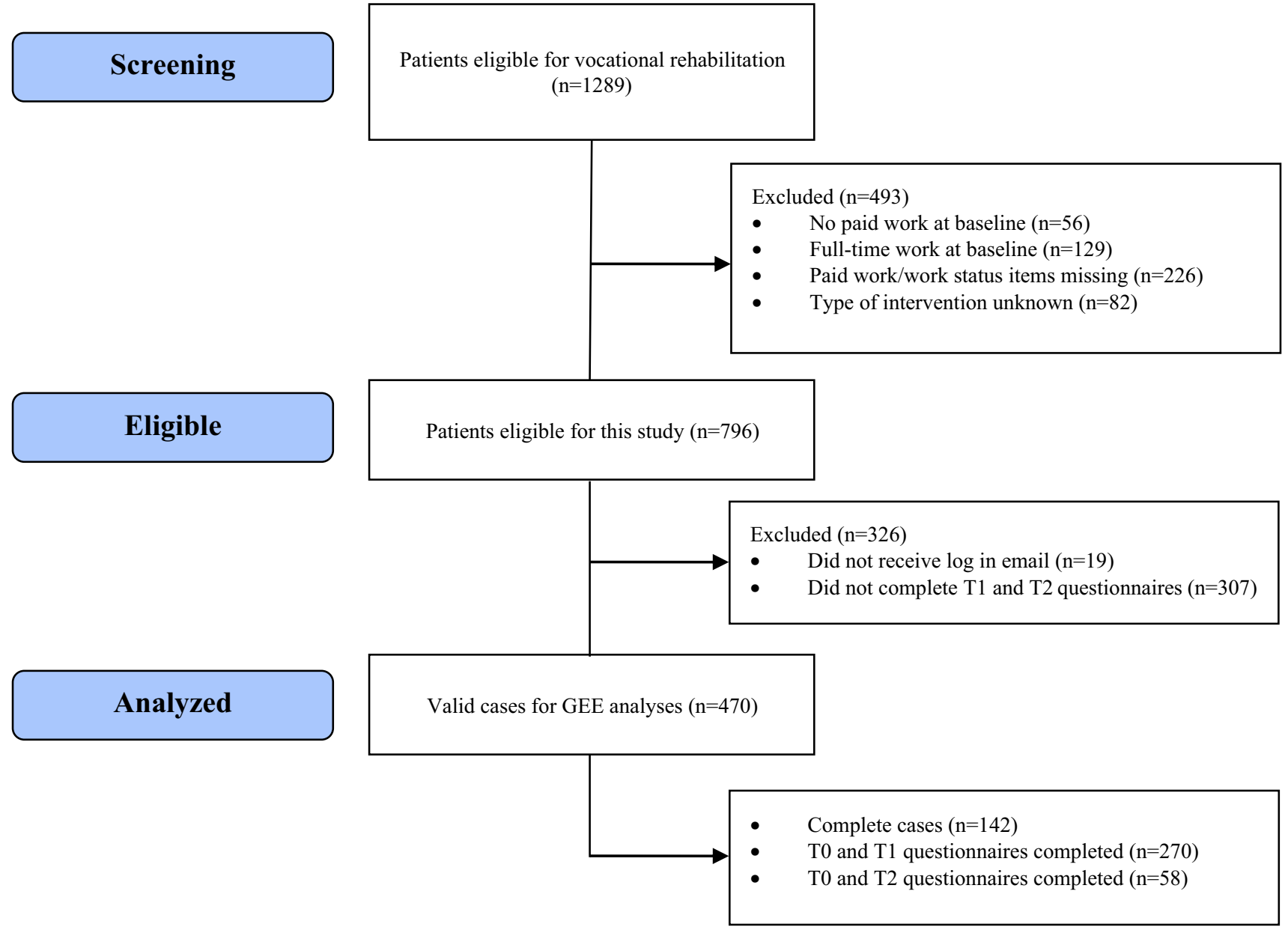

Fig. 1 Flow chart of participants in this study. $T 0$ screening, $T 1$ discharge, $T 2$ 6-months follow-up, GEE generalized estimating equations 


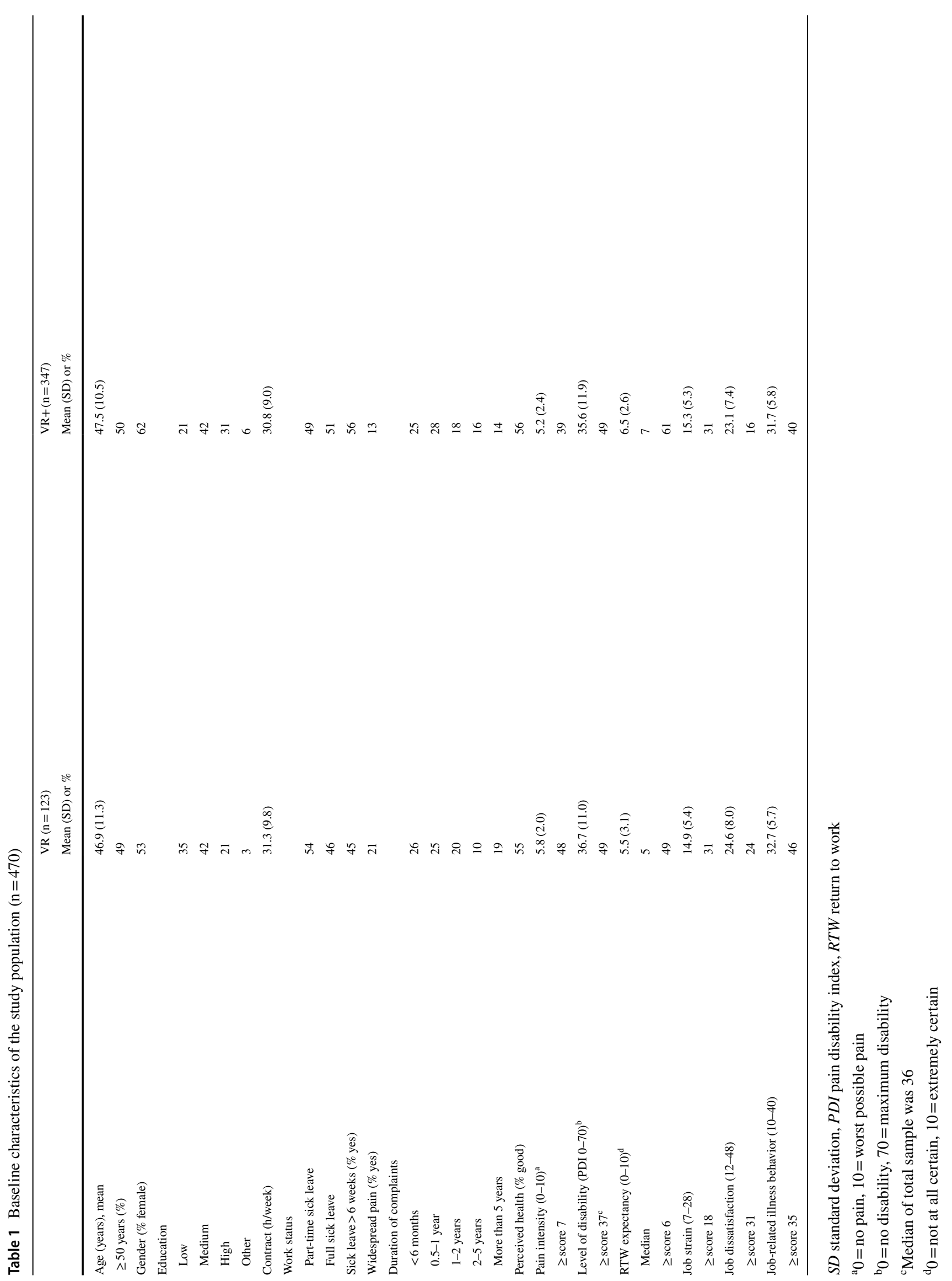




\section{Missing Data}

The missing data mechanism (i.e., missing complete at random [MCAR] or missing at random [MAR] [53]) was analyzed by conducting a T-test and Little MCAR tests.

\section{Results}

Out of 796 eligible patients, a total of 470 (59\%) patient-data was eligible for analyses. Of these, 123 (26\%) received VR and $347(74 \%) \mathrm{VR}+$. Figure 1 shows a flowchart of the participant inclusion and reasons for dropout. The missing data mechanism for T1 and T2 was missing at random. The sample characteristics of both programs are presented in Table 1 .

\section{Work Participation}

At discharge from vocational rehabilitation, $64 \%$ of participants in the VR program and $74 \%$ in the $\mathrm{VR}+$ program achieved work participation (Fig. 2). At 6-months followup, $86 \%$ of participants in the VR program and $87 \%$ in the VR+ program achieved work participation. From baseline to 6-months follow-up, work participation increased $32 \%$ in VR and $38 \%$ in VR+.

\section{GEE Analyses}

The crude model showed a significant longitudinal relationship between VR+ on the achievement of work participation: OR 1.8 (CI 1.2-2.6), $\mathrm{p}=0.01$. The model with time and the interaction intervention*time showed a borderline non-significant relationship between $\mathrm{VR}+$ on the achievement of work participation on discharge: OR 1.8 (CI 1.0-3.4), $\mathrm{p}=0.06$, and a significant relationship on 6-months follow-up: $\mathrm{OR}=1.8$ (CI 1.1-2.8), $\mathrm{p}=0.01$. RTW expectation, age, and gender showed to be confounders in step three of the analyses. The final model (Table 2), with the three confounding variables included, showed a significant relationship between $\mathrm{VR}+$ on the achievement of work participation on 6-months follow-up: OR $=1.7$ (CI 1.0-2.8), $\mathrm{p}=0.04$. A non-significant relationship was observed on discharge: $\mathrm{OR}=1.3$ (CI 0.6-2.5), $\mathrm{p}=0.51$. RTW expectation was the only significant confounder in the final model: OR 3.1 (CI 2.1-4.6), $\mathrm{p}=0.00$ (Table 2).

\section{Discussion}

We hypothesized that patients who received VR with an additional work module (VR+) would have greater odds of achieving work participation compared to patients who
Vocational rehabilitation

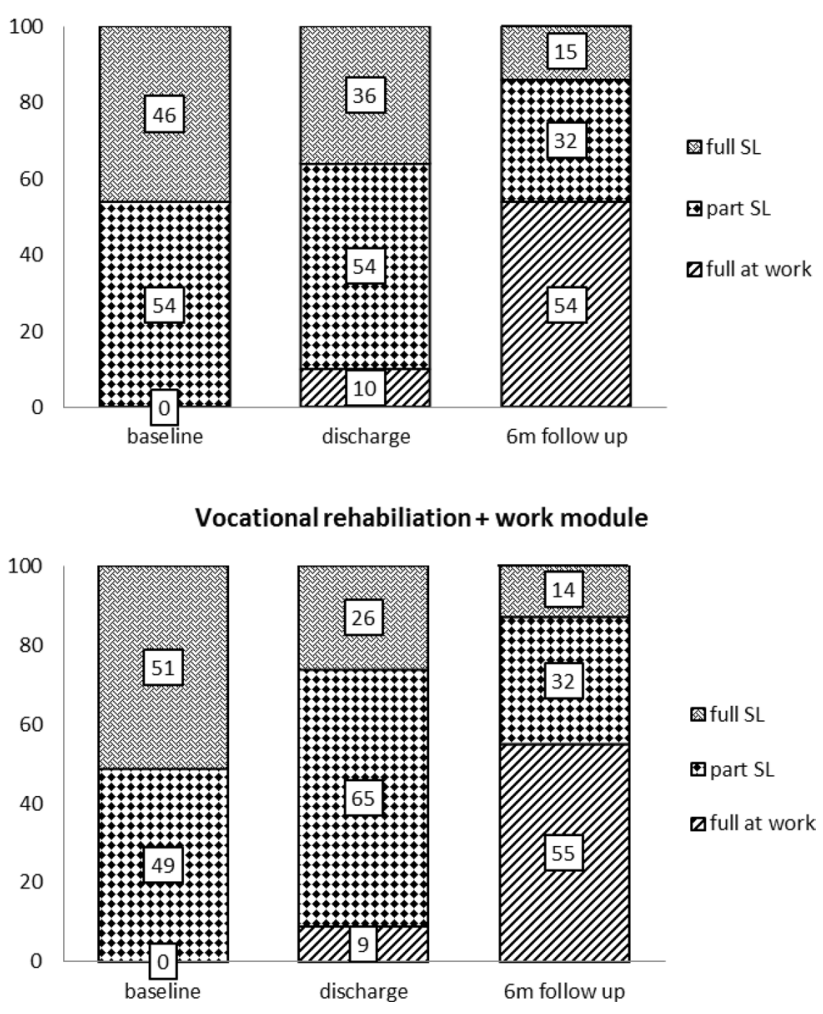

Fig. 2 Working status proportions at baseline, discharge, and 6-months follow-up. $S L$ sick leave. Note Due to rounding features, the total proportion per time point might deviate from $100 \%$

received VR without work module. Because this study demonstrated greater odds of VR+ on work participation at 6-months follow-up, our hypothesis was supported. This finding is consistent with the recommendations reported in systematic reviews to include work components to optimize work participation $[5,6,8,9,17,54,55]$. Both VR programs studied in this paper showed highly beneficial work participation rates at 6-months follow-up: $86 \%$ of the VR group were at work (full-time or part-time) and $87 \%$ of the $\mathrm{VR}+$ group were at work. The work participation rates at 6-months follow-up reported in this paper are higher compared to multi-domain VR described by others, who showed mean work participation proportions of $65 \% \pm 11 \%$ [56-62]. The high and quite similar work participation rates at 6-months follow-up might be a consequence of the Dutch social security system. Within this system, the employer has a mandatory role in offering modified work. All patients in this study had been offered this in some form, including those in the VR group. In Dutch practice, therefore, the contrast between VR and VR+ may have been smaller than in non-Dutch practice. The dosage of VR+ was similar to VR (100 and $90 \mathrm{~h}$ respectively), which may have also contributed to the small difference in results. The difference at discharge was non-significant, which we hypothesized 


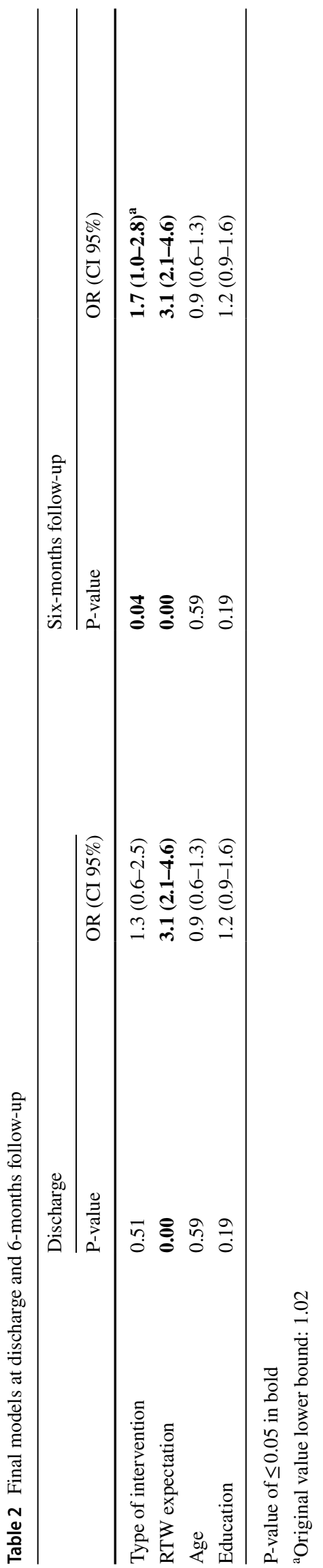

was due to insufficient 'wash-in' of work module effects at discharge. Moreover, the results of this study may provide confirmation that work modifications can support the work reintegration of patients with CMP and sick leave from work [5]. How the three core elements (Box 1) should be delivered optimally, however, may depend on country-specific system characteristics and further study.

The present study was performed within clinical practice and studied the impact of work participation of two multidomain programs. Other studies were conducted in a controlled setting, and frequently compared multi-domain programs with single-component programs or care as usual [5, 6,8-10]. This complicates comparison of the findings of the present study with these studies. Nonetheless, we detected two quite similar studies compared to ours. The first study was an RCT conducted in Norway in patients with neck and back pain. This study showed deviating results compared to our study, namely no significant difference between a group who took part in a multidisciplinary program that included a work focus and a control group who only took part in a multidisciplinary program [15]. One explanation for these results might be the fact that for the multidisciplinary workfocused group it was not possible to intervene at the workplace due to regulations in Norway. The other quite similar retrospective cohort study was conducted in Canada, and showed that patients who completed a multimodal pain program that included RTW coordination had 3.4 higher odds of returning to work compared with patients who received the multimodal program without RTW coordination [16]. However, this study did not correct for RTW expectancy. Based on the present study, and many others [27-30, 37, 45-47], it is clear that RTW expectation is an important confounder in the relationship between an intervention program and a focus on improving work participation. Consequently, a clinical implication is to take RTW expectations into account at inclusion/screening before the start of an interdisciplinary VR program: patients with positive RTW expectations have higher odds of responding successfully to VR or VR+. Low RTW expectations may need further clinical attention to the underlying reason for this, and consequently, targeted intervention if modifiable.

\section{Strengths and Limitations}

One strength of a retrospective study is its observational character, as the researcher is able to observe what actually happens or naturally occurs in practice. This is a great advantage in terms of adaptation for professionals. In addition, in our case, it was possible to correct for confounding variables which were clustered a priori based on the biopsychosocial model. This increases knowledge of which factors are important to take into account in research and clinical practice. Furthermore, because we applied GEE analyses, it 
was possible to include the dependency of in-person change in work participation over time in the statistical model. This increased the robustness of our findings.

One limitation of a retrospective cohort design is that the intended intervention is less controllable, which may bias the results. In our case, contamination bias between the two programs could have occurred. Patients from both intervention groups were undertaking rehabilitation together. Patients who only participated in the VR program probably obtained information from patients who completed the VR+ program and from the RTW coordinators during group meetings or coffee breaks. Because 3 out of 4 patients received the $\mathrm{VR}+$ program, the chance of contamination bias, resulting in a lack of contrast, was high.

Another limitation of this study was that regarding the $\mathrm{VR}+$ program it was unknown if patient actually received the components of the work module (ergonomic adjustments, case management, RTW plan) in practice. Compliance with the work module could have influenced work participation, but we were unfortunately not able to control for this factor.

Selection bias may also have occurred, as the type of program a patient participated in was dependent on the employer's willingness to pay for the additional work module. However, at baseline there were no substantial differences between job dissatisfaction and job strain between the VR+ and VR groups. There were probably other factors which influenced the outcomes of the additional work module. From the beginning, one might expected that the $\mathrm{VR}+$ group would have higher odds of achieving work participation compared to the VR group, due to differences in a number of variables: the VR group was less educated, had a higher proportion of widespread pain, higher pain scores, higher disability scores, and lower RTW expectancy. However, almost all of the independent (possible confounding) variables selected a priori were not included or did not contribute to the final models. The only significant independent variable (and also confounder) in the final models at discharge and 6-months follow-up was RTW expectation. Because a positive association of VR+ and work participation at 6-months follow-up in the final model was found, we proposed the influence of selection bias on our results was low.

One final limitation was the proportion of loss to followup (41\%), which negatively influenced the sample size of the analyzed cases $(n=470)$. This might hampered the sample's representativeness with the population of interest. Post-hoc analyses with the eligible source population of $n=796$ showed similar meaningful baseline characteristics compared to the analyzed cases (age, gender RTW expectancy, pain intensity, pain disability, sick leave status). Hence, we assume our findings were representative for the population of interest.

\section{Methodological Considerations}

One methodological consideration is that we included RTW expectation as a factor in the GEE model, but we did not include "work environment" as a factor in the GEE model. It is however known that when subjects return to a workplace with a bad environment, they are more likely to fall back into sick leave again [27]. It may be hypothesized that the RTW expectation variable is not solely an individual variable, but that this variable is influenced by workplace specific variables, such as job strain and work environment. Because we did not include the latter factor in the GEE model, this might have overestimated the relationship between RTW expectation and work participation. Another methodological consideration is the high proportion of incomplete cases (i.e. only valid data on baseline-discharge or baseline-6mo follow-up; Fig. 2) which were used for the GEE analyses. However, one of the advantages of the GEE approach is that it can handle missing data very well and that data imputation techniques are not necessary to produce robust results [63].

\section{Clinical Implications}

The results of this study suggest to add in general a work module to VR in order to optimize work participation of patients with chronic musculoskeletal pain and sick leave from work. When actions for improving the work participation are already accomplished at the workplace before entering VR, it can be considered not to add a work module to VR. This should be discussed on a patient bases at the inclusion/screening phase before VR starts.

\section{Future Directions}

Because our study showed that patients with positive RTW expectations had three times higher odds of responding successfully after VR (independent of type of program), we recommend that future research should assess RTW expectations at baseline and correct for this variable during the analyses. Another future direction for research would be to execute return on investment analyses on the added value of work modules when nested in VR. This information is important for those who are asked to reimburse these modules.

\section{Conclusion}

There is a positive and significant longitudinal relationship between vocational rehabilitation with additional work module, compared to VR without additional work module, on work participation of patients with chronic musculoskeletal pain and sick leave from work. 
Funding No commercial sponsorship was involved in designing or conducting the study.

\section{Compliance with Ethical Standards}

Conflict of interest All authors declare that they have no conflict of interest.

Ethical Approval All procedures performed were in accordance with the ethical standards of the institutional research committee and with the 1964 Helsinki declaration and its later amendments or comparable ethical standards. The Medical Ethical Committee of the Academic Medical Center, Amsterdam, the Netherlands, authorized this study and decided that a full application was not required.

Informed Consent Informed consent was obtained from all individual participants included in the study.

Open Access This article is licensed under a Creative Commons Attribution 4.0 International License, which permits use, sharing, adaptation, distribution and reproduction in any medium or format, as long as you give appropriate credit to the original author(s) and the source, provide a link to the Creative Commons licence, and indicate if changes were made. The images or other third party material in this article are included in the article's Creative Commons licence, unless indicated otherwise in a credit line to the material. If material is not included in the article's Creative Commons licence and your intended use is not permitted by statutory regulation or exceeds the permitted use, you will need to obtain permission directly from the copyright holder. To view a copy of this licence, visit http://creativecommons.org/licenses/by/4.0/.

\section{References}

1. Breivik H, Collett B, Ventafridda V, Cohen R, Gallacher D. Survey of chronic pain in Europe: prevalence, impact on daily life, and treatment. Eur J Pain. 2006;10(4):287-333.

2. Koke AJ, Smeets RJ, Schreurs KM, van Baalen B, de Haan P, Remerie SC, et al. Dutch Dataset Pain Rehabilitation in daily practice: content, patient characteristics and reference data. Eur J Pain. 2017;21(3):434-444.

3. Aronsson G, Gustafsson K, Dallner M. Sick but yet at work. An empirical study of sickness presenteeism. J Epidemiol Commun Health. 2000;54(7):502-509.

4. OECD. Sickness, disability and work: breaking the barriers. A synthesis of findings across OECD countries [report]. Paris: OECD; 2010.

5. Cullen KL, Irvin E, Collie A, Clay F, Gensby U, Jennings PA, et al. Effectiveness of workplace interventions in return-to-work for musculoskeletal, pain-related and mental health conditions: an update of the evidence and messages for practitioners. J Occup Rehabil. 2018;28(1):1-15.

6. Palmer KT, Harris EC, Linaker C, Barker M, Lawrence W, Cooper $\mathrm{C}$, et al. Effectiveness of community- and workplace-based interventions to manage musculoskeletal-related sickness absence and job loss: a systematic review. Rheumatology (Oxford). 2012;51(2):230-242.

7. Waddell G, Burton AK, Kendall NAS. Vocational rehabilitation: what works, for whom, and when?. London: TSO; 2013.
8. Waterschoot FP, Dijkstra PU, Geertzen JH, Reneman MF. Dose or content? Effectiveness of pain rehabilitation programs for patients with chronic low back pain: a systematic review. Aurthor reply. Pain. 2014;155(9):1902-1903.

9. van Vilsteren M, van Oostrom SH, de Vet HC, Franche RL, Boot CR, Anema JR. Workplace interventions to prevent work disability in workers on sick leave. Cochrane Database Syst Rev. 2015;10:CD006955.

10. Kamper SJ, Apeldoorn AT, Chiarotto A, Smeets RJ, Ostelo RW, Guzman J, et al. Multidisciplinary biopsychosocial rehabilitation for chronic low back pain. Cochrane Database Syst Rev. 2014;9:CD000963.

11. Loisel P, Gosselin L, Durand P, Lemaire J, Poitras S, Abenhaim L. Implementation of a participatory ergonomics program in the rehabilitation of workers suffering from subacute back pain. Appl Ergon. 2001;32(1):53-60.

12. Anema JR, Cuelenaere B, van der Beek AJ, Knol DL, de Vet HC, van Mechelen W. The effectiveness of ergonomic interventions on return-to-work after low back pain; a prospective two year cohort study in six countries on low back pain patients sicklisted for 3-4 months. Occup Environ Med. 2004;61(4):289-294.

13. Anema JR, Steenstra IA, Bongers PM, de Vet HC, Knol DL, Loisel P, et al. Multidisciplinary rehabilitation for subacute low back pain: graded activity or workplace intervention or both? A randomized controlled trial. Spine (Phila PA 1976). 2007;32(3):291-298.

14. Loisel P, Lemaire J, Poitras S, Durand MJ, Champagne F, Stock $\mathrm{S}$, et al. Cost-benefit and cost-effectiveness analysis of a disability prevention model for back pain management: a six year follow up study. Occup Environ Med. 2002;59(12):807-815.

15. Myhre K, Marchand GH, Leivseth G, Keller A, Bautz-Holter E, Sandvik L, et al. The effect of work-focused rehabilitation among patients with neck and back pain: a randomized controlled trial. Spine (Phila PA 1976). 2014;39(24):1999-2006.

16. Hamer H, Gandhi R, Wong S, Mahomed NN. Predicting return to work following treatment of chronic pain disorder. Occup Med (Lond). 2013;63(4):253-259.

17. Schaafsma F, Schonstein E, Whelan KM, Ulvestad E, Kenny DT, Verbeek JH. Physical conditioning programs for improving work outcomes in workers with back pain. Cochrane Database Syst Rev. 2010;37(1):CD001822.

18. von Elm E, Altman DG, Egger M, Pocock SJ, Gotzsche PC, Vandenbroucke JP. The strengthening the reporting of observational studies in epidemiology (STROBE) statement: guidelines for reporting observational studies. J Clin Epidemiol. 2008;61(4):344-349.

19. Beemster T, van Bennekom C, van Velzen J, Reneman M, FringsDresen $\mathrm{M}$. The interpretation of change score of the pain disability index after vocational rehabilitation is baseline dependent. Health Qual Life Outcomes. 2018;16(1):182.

20. The Law on Gate keeping Disability Insurance [Dutch: Wet verbetering poortwachter]. Accessed 22 January 2018. https://www. arboportaal.nl/onderwerpen/wet-verbetering-poortwachter.

21. Beemster TT, van Velzen JM, van Bennekom CA, Frings-Dresen MH, Reneman MF. Cost-effectiveness of 40-hour versus 100-hour vocational rehabilitation on work participation for workers on sick leave due to subacute or chronic musculoskeletal pain: study protocol for a randomized controlled trial. Trials. 2015;16:317.

22. Beemster TT, van Velzen JM, van Bennekom CAM, Reneman MF, Frings-Dresen MHW. Test-retest reliability, agreement and responsiveness of productivity loss (iPCQ-VR) and healthcare utilization (TiCP-VR) Questionnaires for sick workers with chronic musculoskeletal pain. J Occup Rehabil. 2018;29:91-103. 
23. Waddel G, Burton AK. Concepts of rehabilitation for the management of common health problems. London: The Stationery Office; 2004.

24. Reneman MF, Beemster TT, Edelaar MJ, van Velzen JM, van Bennekom C, Escorpizo R. Towards an ICF- and IMMPACT-based pain vocational rehabilitation core set in the Netherlands. J Occup Rehabil. 2013;23(4):576-584.

25. Boonstra AM, Reneman MF, Waaksma BR, Schiphorst Preuper HR, Stewart RE. Predictors of multidisciplinary treatment outcome in patients with chronic musculoskeletal pain. Disabil Rehabil. 2015;37(14):1242-1250.

26. Oyeflaten I, Lie SA, Ihlebaek CM, Eriksen HR. Prognostic factors for return to work, sickness benefits, and transitions between these states: a 4-year follow-up after work-related rehabilitation. J Occup Rehabil. 2014;24(2):199-212.

27. Selander J, Marnetoft SU, Bergroth A, Ekholm J. Return to work following vocational rehabilitation for neck, back and shoulder problems: risk factors reviewed. Disabil Rehabil. 2002;24(14):704-712.

28. Steenstra IA, Munhall C, Irvin E, Oranye N, Passmore S, Van Eerd D, et al. Systematic review of prognostic factors for return to work in workers with sub acute and chronic low back pain. J Occup Rehabil. 2017;27(3):369-381.

29. Opsahl J, Eriksen HR, Tveito TH. Do expectancies of return to work and Job satisfaction predict actual return to work in workers with long lasting LBP? BMC Musculoskelet Disord. 2016;17(1):481.

30. Volker D, Zijlstra-Vlasveld MC, Brouwers EP, van Lomwel AG, van der Feltz-Cornelis CM. Return-to-work self-efficacy and actual return to work among long-term sick-listed employees. J Occup Rehabil. 2015;25(2):423-431.

31. Fliesser M, De Witt HJ, Wippert PM. The choice that matters: the relative influence of socioeconomic status indicators on chronic back pain-a longitudinal study. BMC Health Serv Res. 2017;17(1):800.

32. Haveraaen LA, Skarpaas LS, Berg JE, Aas RW. Do psychological job demands, decision control and social support predictreturn to work three months after a return-to-work (RTW) programme? The rapid-RTW cohort study. Work. 2015;53(1):61-71.

33. Kaikkonen R, Harkanen T, Rahkonen O, Gould R, Koskinen S. Explaining educational differences in sickness absence: a population-based follow-up study. Scand J Work Environ Health. 2015;41(4):338-346.

34. Vendrig A. De vragenlijst arbeidsreïntegratie. Diagnostiek-Wijzer. 2005;8:27-39.

35. Vendrig L, van Hove M, van Meijel M, Donceel P. Voorspellen van de verwachte verzuimduur met de Vragenlijst ArbeidsReïntegratie (VAR) [Predicting the expected duration of sickness absence by using the Questionnaire Work Resumption]. TBV. 2011;19(1):7-13.

36. Asih S, Neblett R, Mayer TG, Gatchel RJ. Does the length of disability between injury and functional restoration program entry affect treatment outcomes for patients with chronic disabling occupational musculoskeletal disorders? J Occup Rehabil. 2018;28(1):57-67.

37. Besen E, Young AE, Shaw WS. Returning to work following low back pain: towards a model of individual psychosocial factors. J Occup Rehabil. 2015;25(1):25-37.

38. van der Zee-Neuen A, Putrik P, Ramiro S, Keszei A, de Bie R, Chorus A, et al. Work outcome in persons with musculoskeletal diseases: comparison with other chronic diseases \& the role of musculoskeletal diseases in multimorbidity. BMC Musculoskelet Disord. 2017;18(1):10.

39. Storheim K, Brox JI, Holm I, Bo K. Predictors of return to work in patients sick listed for sub-acute low back pain: a 12-month follow-up study. J Rehabil Med. 2005;37(6):365-371.
40. van Hooff ML, Spruit M, O’Dowd JK, van Lankveld W, Fairbank JC, van Limbeek J. Predictive factors for successful clinical outcome 1 year after an intensive combined physical and psychological programme for chronic low back pain. Eur Spine J. 2014;23(1):102-112.

41. Leveille SG, Bean J, Ngo L, McMullen W, Guralnik JM. The pathway from musculoskeletal pain to mobility difficulty in older disabled women. Pain. 2007;128(1-2):69-77.

42. Pollard CA. Preliminary validity study of the pain disability index. Percept Mot Skills. 1984;59(3):974.

43. Hays RD, Morales LS. The RAND-36 measure of health-related quality of life. Ann Med. 2001;33(5):350-357.

44. Van der Zee K, Sanderman R. Measuring general health status with the RAND-36. Users manual [Het meten van de algemene gezondheidstoestand met de RAND-36. Een handleiding]. 2nd edition. Groningen, the Netherlands: Research Institute SHARE; 2012; https://www.umcg.nl/.

45. Black O, Keegel T, Sim MR, Collie A, Smith P. The effect of self-efficacy on return-to-work outcomes for workers with psychological or upper-body musculoskeletal injuries: a review of the literature. J Occup Rehabil. 2018;28(1):16-27.

46. Ebrahim S, Malachowski C, Kamaleldin M, Mulla SM, Montoya L, Bance S, et al. Measures of patients' expectations about recovery: a systematic review. J Occup Rehabil. 2015;25(1):240-255.

47. Hallegraeff JM, Krijnen WP, van der Schans CP, de Greef MH. Expectations about recovery from acute non-specific low back pain predict absence from usual work due to chronic low back pain: a systematic review. J Physiother. 2012;58(3):165-172.

48. Reis RJ, Utzet M, La Rocca PF, Nedel FB, Martin M, Navarro A. Previous sick leaves as predictor of subsequent ones. Int Arch Occup Environ Health. 2011;84(5):491-499.

49. Roelen CA, Koopmans PC, Schreuder JA, Anema JR, van der Beek AJ. The history of registered sickness absence predicts future sickness absence. Occup Med (Lond). 2011;61(2):96-101.

50. Viikari-Juntura E, Virta LJ, Kausto J, Autti-Ramo I, Martimo KP, Laaksonen M, et al. Legislative change enabling use of early parttime sick leave enhanced return to work and work participation in Finland. Scand J Work Environ Health. 2017;43(5):447-456.

51. Stapelfeldt CM, Christiansen DH, Jensen OK, Nielsen CV, Petersen KD, Jensen C. Subgroup analyses on return to work in sick-listed employees with low back pain in a randomised trial comparing brief and multidisciplinary intervention. BMC Musculoskelet Disord. 2011;12:112.

52. Dekkers-Sanchez PM, Hoving JL, Sluiter JK, Frings-Dresen MH. Factors associated with long-term sick leave in sicklisted employees: a systematic review. Occup Environ Med. 2008;65(3):153-157.

53. Sterne JA, White IR, Carlin JB, Spratt M, Royston P, Kenward MG, et al. Multiple imputation for missing data in epidemiological and clinical research: potential and pitfalls. BMJ. 2009;338:b2393.

54. Hoefsmit N, Houkes I, Nijhuis FJ. Intervention characteristics that facilitate return to work after sickness absence: a systematic literature review. J Occup Rehabil. 2012;22(4):462-477.

55. Meijer EM, Sluiter JK, Frings-Dresen MH. Evaluation of effective return-to-work treatment programs for sick-listed patients with non-specific musculoskeletal complaints: a systematic review. Int Arch Occup Environ Health. 2005;78(7):523-532.

56. Bethge M, Herbold D, Trowitzsch L, Jacobi C. Work status and health-related quality of life following multimodal work hardening: a cluster randomised trial. J Back Musculoskelet Rehabil. 2011;24(3):161-172.

57. Brendbekken R, Eriksen HR, Grasdal A, Harris A, Hagen EM, Tangen T. Return to work in patients with chronic musculoskeletal pain: multidisciplinary intervention versus brief intervention: a randomized clinical trial. J Occup Rehabil. 2017;27(1):82-91. 
58. Harris A, Moe TF, Eriksen HR, Tangen T, Lie SA, Tveito TH, et al. Brief intervention, physical exercise and cognitive behavioural group therapy for patients with chronic low back pain (The CINS trial). Eur J Pain. 2017;21(8):1397-1407.

59. Jousset N, Fanello S, Bontoux L, Dubus V, Billabert C, Vielle $\mathrm{B}$, et al. Effects of functional restoration versus 3 hours per week physical therapy: a randomized controlled study. Spine (Phila PA 1976). 2004;29(5):487-493.

60. Linton SJ, Boersma K, Traczyk M, Shaw W, Nicholas M. Early workplace communication and problem solving to prevent back disability: results of a randomized controlled trial among high-risk workers and their supervisors. J Occup Rehabil. 2016;26(2):150-159.

61. Skouen JS, Grasdal AL, Haldorsen EM, Ursin H. Relative costeffectiveness of extensive and light multidisciplinary treatment programs versus treatment as usual for patients with chronic low back pain on long-term sick leave: randomized controlled study. Spine (Phila PA 1976). 2002;27(9):901-909.

62. Henchoz Y, de Goumoens P, So AK, Paillex R. Functional multidisciplinary rehabilitation versus outpatient physiotherapy for non specific low back pain: randomized controlled trial. Swiss Med Wkly. 2010;140:w13133.

63. Twisk J, de Vente W. Attrition in longitudinal studies How to deal with missing data. J Clin Epidemiol. 2002;55(4):329-337.

Publisher's Note Springer Nature remains neutral with regard to jurisdictional claims in published maps and institutional affiliations. 